\title{
NIETZSCHE, EL SOBREHUMANO Y EL TRANSHUMANISMO ${ }^{12}$
}

\section{NIETZSCHE, THE OVERHUMAN AND TRANSHUMANISM}

\author{
Stefan Lorenz Sorgner ${ }^{3}$ \\ John Cabot University/Italy \\ Recibido: 17/07/2016 - Aprobado: 16/09/2017
}

\begin{abstract}
Resumen
Bostrom niega a Nietzsche como un ancestro del movimiento transhumanista, en tanto alega que hay solo algunas "similitudes a nivel superficial con la visión de Nietzsche" (Bostrom, $2005^{a}$, p.4). Por el contrario, yo pienso que se pueden hallar similitudes significativas entre lo posthumano y el sobrehumano, a un nivel fundamental. Además, creo que Nietzsche explica la relevancia del sobrehumano al referirse a una dimensión que parece carecer de transhumanismo. Para explicar mi posición, avanzaré de la siguiente manera. Primero, compararé el concepto de posthumano con el de sobrehumano de Nietzsche, concentrándome más en sus similitudes que en sus diferencias. En segundo lugar, contextualizaré el sobrehumano en la visión general de Nietzsche, de modo que pueda señalar cuál dimensión me parece que carece de pensamiento transhumanista.
\end{abstract}

Palabras clave: posthumano, transhumano, sobrehumano, Nietzsche

\section{Summary}

Bostrom rejects Nietzsche as an ancestor of the transhumanist movement, as he claims that there were merely some "surface-level similarities with the Nietzschean vision" (Bostrom 2005a, 4). In contrast to Bostrom, I think that significant similarities between the posthuman and the overhuman can be found on a fundamental level. In addition, it seems to me that Nietzsche explained the relevance of the overhuman by referring to a

1 Este artículo apareció originalmente en Journal of Evolution and Technology, Vol. 20 Issue 1 - March 2009 págs. 29-42. http://jetpress.org/v20/sorgner.htm

2 Traducción de Jonathan Piedra Alegría, quien agradece la gentileza del Dr. Stefan Lorenz Sorgner por permitirle la traducción y publicación de este texto.

3 Director de la red Beyond Humanism Network, Fellow del Institute for Ethics and Emerging Technologies y Research Fellow en el Ewha Institute for the Humanities at Ewha Womans University/Seoul, Korea. 
dimension which seems to be lacking in transhumanism. In order to explain my position, I will progress as follows. First, I will compare the concept of the posthuman to that of Nietzsche's overhuman, focusing more on their similarities than their differences. Second, I will contextualise the overhuman in Nietzsche's general vision, so that I can point out which dimension seems to me to be lacking in transhumanist thought.

Key words: posthuman, transhuman, overhuman, Nietzsche.

Inicialmente, cuando me estaba familiarizando con el movimiento transhumanista, pensé que había muchas similitudes fundamentales entre el transhumanismo y la filosofía de Nietzsche, especialmente en lo relacionado con el concepto del posthumano y el sobrehumano ${ }^{4}$ (overhuman) de Nietzsche. Pues bien, esto es precisamente lo que quiero mostrar en este artículo. Estoy empleando el término sobrehumano (overhuman) en lugar de superhombre (overman), ya que en alemán el término Übermensch puede aplicarse a ambos sexos. La palabra sobrehumano los puede incluir, mientras que superhombre no lo hace.

He descubierto que Bostrom, un líder transhumanista, rechaza a Nietzsche como un antecedente del movimiento transhumanista, ya que él afirma que no hay más que algunas «similitudes a nivel superficial con la visión nietzscheana"5 (Bostrom, 2005a, p. 4).

En contraste con Bostrom, creo que hay similitudes significativas entre el posthumano y el sobrehumano que pueden ser encontradas en un nivel fundamental. Habermas estaría de acuerdo conmigo en este sentido, puesto que él ya se ha referido a las similitudes de estos dos pensamientos.

4 Traducimos overhuman como sobrehumano, para ser fieles al sentido que el autor quiere darle a esta palabra. Por el contrario, en el caso de Übermensch seguimos manejando la traducción más utilizada de este concepto en español (superhombre) por un asunto de conveniencia y costumbre, aunque no necesariamente por un tema de precisión filosófica, ya que la proposición Über hace referencia a algo sobre de o encima de e inclusive otro lado de, dependiendo del contexto y la utilización. Nos parece que Über en muchas ocasiones no se comprende correctamente desde la palabra súper en español, que generalmente se usa como una forma de magnificación de un ente, estado o situación. Esto, igualmente, es lo que provoca en algunas ocasiones apropiaciones muy inadecuadas o interpretaciones poco relacionadas con lo que Nietzsche proponía con el Übermensch. El superhombre no es un ser humano promedio potenciado. Es, para resumirlo de una manera muy simplificadora, aquel ser humano (que según Nietzsche todavía no existe) que tiene como características principales: 1. la afirmación no-dialéctica de la existencia y 2. la creación de valores (de manera que se relaciona con la crítica moral de Nietzsche, así como con la transmutación de los valores) y que, por lo tanto, implica la energía afirmativa de la vida. En el caso del autor del artículo, este cambia superhombre por sobrehumano, por un asunto de inclusión de género, no de resignificación semántica necesariamente. N. del T.

5 Al igual que el documento que aquí se presenta, las citas bibliográficas que aparecen en el texto han sido traducidas de la versión original de este artículo. N. del. T 
Habermas parece considerar, sin embargo, absurdas ambas posturas. Por lo menos, se puede decir que se refiere a los transhumanistas como un grupo de intelectuales locos que afortunadamente no han logrado el apoyo de un grupo más grande de seguidores para sus visiones elitistas (Habermas, 2001). ${ }^{6}$

Me parece, además, que Nietzsche explicó la relevancia del sobrehumano haciendo referencia a una dimensión que parece faltar en el transhumanismo. Con el fin de explicar mi posición, desarrollaré mis ideas de la siguiente manera. En primer lugar, voy a comparar el concepto del posthumano con el sobrehumano de Nietzsche, centrándome más en sus similitudes que en sus diferencias. En segundo lugar, voy a contextualizar el sobrehumano en la visión general de Nietzsche, de modo que pueda señalar cuál es la dimensión de la que, a mi parecer, carece el pensamiento transhumanista.

\section{El posthumano y el sobrehumano de Nietzsche}

Primero, me centraré directamente en la comparación entre el posthumano y sobrehumano de Nietzsche. Para esto trataré con algunos de los principios fundamentales, en donde el concepto del posthumano se puede encontrar en la versión de Bostrom del transhumanismo, y los principios correspondientes dentro del pensamiento de Nietzsche. Luego haré una breve comparación entre los puntos de vista dinámicos de la naturaleza y los valores, y sus posiciones relacionadas con la naturaleza humana, la mejora, la educación, la revalorización de los valores y la evolución hacia una especie superior.

\section{La evolución de la naturaleza humana y los valores.}

En primer lugar, tanto los transhumanistas como Nietzsche tienen una visión dinámica de la naturaleza y los valores. Bostrom menciona que "los transhumanistas ven la naturaleza humana como un trabajo en proceso" (2005b, p. 1). Pues lo mismo ocurre con Nietzsche, ya que él lleva a cabo una dinámica de la metafísica de la voluntad de poder que se aplica a todos los seres, incluyendo a los seres humanos, lo que implica que todas las cosas

6 El estudioso británico sobre Nietzsche, Ansell-Pearson (1997), simplemente reconoce algunas similitudes entre Nietzsche y el transhumanismo. 
están constantemente experimentando el cambio. ${ }^{7}$ No hay nada que esté fijo (fixed) eternamente. Según Nietzsche, los seres humanos son organismos constituidos a partir de quantums ${ }^{8}$ individuales de poder o de constelaciones de voluntad de poder. Este concepto se puede aclarar haciendo referencia a la monadología ${ }^{9}$ de Leibniz. Un quantum de energía es una sola entidad como una mónada. En contraste con la mónada, este puede interactuar con otros quantums de energía que pueden crecer, nutrirse ellos mismos (de manera metafórica) y que tienen un punto de vista sobre el mundo. Esta perspectiva habilita al quantum para decidir qué hacer a continuación, lo que depende de sus opciones y de su concepción del poder. En cuanto a la noción de poder, esta se emplea de una manera extremadamente amplia y abierta. En cada estado en el que un quantum de poder es más fuerte, más capaz que otro y tiene el potencial de dominar al otro, representa un estado de poder.

Según Nietzsche, todas las entidades están constituidas a partir de estas constelaciones de poder. La dinámica del poder también es la base del proceso de la evolución que es responsable de ocasionar a la especie humana, los animales y las plantas. Todos los organismos llegaron a existir porque las condiciones que provocaron a los respectivos organismos eran el mejor medio posible para la realización de la lucha por el poder de los organismos anteriores. Es así como, eventualmente, los seres humanos llegaron a existir.

Sin embargo, la especie "ser humano", como todas las especies, no es eternamente fija e inmutable. Entró en la existencia, puede desaparecer de ella y puede evolucionar en una especie diferente. Los miembros individuales de una especie solo tienen un cierto potencial limitado que se encuentra restringido por su pertenencia a una especie específica. Cada especie representa a una especie no solo porque se trata de una comunidad cuyos miembros tienen el potencial de reproducirse entre sí, sino también porque sus miembros tienen ciertos límites.

Un ser humano como "ser humano" solo tiene un potencial limitado, al igual que sus capacidades, ya que él pertenece a la especie humana y cualquier especie se define por sus límites. Ninguna especie puede ir más allá de sus

7 En los siguientes párrafos voy a resumir mi lectura de la metafísica de la voluntad de poder de Nietzsche (Sorgner, 2007).

8 En física, el quanto (o cuanto) se refiere al valor mínimo que puede tomar una cierta magnitud en un sistema. N. del T.

9 Cf. Sorgner, 2007, p. 50. 
límites. Nietzsche sostiene que si un ser humano ha adquirido capacidades especiales entonces este no puede transmitirlas a sus descendientes. Sin embargo, un cierto tipo de lamarckismo puede ser encontrado en Nietzsche, ya que hace énfasis en que ciertas tendencias se pueden heredar. Si a un hombre le gusta comer bien y disfrutar de la compañía de las mujeres, entonces es aconsejable que su hijo no tenga una vida casta y ascética (Nietzsche, 1967). Si se presenta un cierto estado individual y social que Nietzsche no describe detalladamente, la evolución podría tener lugar y las especies podrían evolucionar-lgo así es mantenido por los transhumanistas-.

Bostrom señala: "Es de común entender que sería ingenuo pensar que la condición humana y la naturaleza humana seguirán siendo más o menos las mismas por mucho más tiempo" (2001. http://www.nickbostrom.com/ tra/values.html).

Nietzsche podría no ser tan optimista como Bostrom, en vista de que él no argumenta que tendrá lugar pronto el progreso evolutivo en el que los seres humanos están involucrados. Sin embargo, acuerda, igual que los transhumanistas, en que eventualmente pasará, si es que la especie humana no deja de existir.

Las dinámicas ontológicas que se pueden encontrar tanto en el transhumanismo como en la filosofía de Nietzsche, también, se pueden aplicar al nivel de los valores. Aquí, Bostrom afirma que:

El transhumanismo es una filosofía dinámica, destinada a evolucionar a medida que se disponga de nueva información o que emerjan nuevos desafíos. Un valor transhumanista es, por lo tanto, cultivar una actitud de cuestionamiento, así como la voluntad de revisar las creencias y las suposiciones propias (2001. http://www.nickbostrom.com/tra/values.html).

Nietzsche estaría de acuerdo en que los valores han sufrido muchos cambios. Su interpretación de la evolución de los valores aparece en el relato de la Genealogía de la moral (1967, pp. 257-289). Los valores están sujetos a cambios en varios niveles: en un nivel social y cultural, así como en uno personal. El concepto de poder de Nietzsche está estrechamente relacionado con el concepto de valor, ya que este puede modificarse teniendo en cuenta las nuevas experiencias y los puntos de vista. El contenido del 
concepto de poder es perspectivo (Sorgner, 2007). No hay valores absolutos e invariables, ya que no hay un reino platónico de las ideas en el que algo podría permanecer fijo.

\section{La ciencia, la mejora, y la educación.}

Nietzsche y los transhumanistas tienen una visión del mundo que se aparta significativamente de la que tiene el cristianismo tradicional o de aquellas visiones que hayan heredado muchos valores cristianos. Como todavía hoy en día se pueden encontrar gran cantidad de elementos del pensamiento cristiano en el sistema de valores de muchas personas, tanto Nietzsche como los transhumanistas están a favor de lograr una revalorización de los valores.

Bostrom enfatiza: "Los transhumanistas insisten en que nuestros preceptos morales, así como las intuiciones recibidas no son en general suficientes para guiar la política" (Bostrom, 2001.http://www.nickbostrom. $\mathrm{com} / \mathrm{tra} / \mathrm{values} . \mathrm{html})$. En consecuencia, sugiere valores que tengan en cuenta una visión dinámica del mundo:

Así que, podemos incluir en esta nuestra lista de valores transhumanistas, la promoción de la comprensión de dónde estamos y hacia dónde nos dirigimos. Este valor encierra otros: el pensamiento crítico, la receptividad, la investigación científica y la discusión abierta como importantes ayudas para aumentar la preparación intelectual de la sociedad (Bostrom, 2001. http:// www.nickbostrom.com/tra/values.html).

Nuevamente Nietzsche estaría de acuerdo. Su respeto por el pensamiento crítico era inmenso -de hecho, es considerado ampliamente como uno de los críticos más duros de la moral y la religión-. Por otra parte, también valoraba enormemente la investigación científica (Sorgner, 2007), a pesar de que su respeto por la ciencia a menudo se ha subestimado. En varios pasajes señala que el futuro será gobernado por un espíritu científico, lo que le hace pensar que mucha gente futura verá a su filosofía como algo plausible, ya que su forma de pensar supone un atractivo para las personas con mentalidad científica. 
La buena concepción que Nietzsche tenía de las ciencias ha sido reconocida por la mayoría de los estudiosos de Nietzsche. ${ }^{10}$ Por ejemplo, su teoría del eterno retorno se basa en premisas que han sido sostenidas por muchos científicos. Su antropología de la voluntad de poder tiene muchas similitudes con las científicas. A pesar de que es crítico de Darwin, también tiene una teoría de la evolución. Nietzsche a menudo es más crítico con los pensadores que están más cerca de su propia comprensión de las cosas. En el caso de Darwin, la crítica de Nietzsche está arraigada principalmente en su concepción de que los seres humanos se esfuerzan únicamente por el poder. Por lo tanto, su concepción implica que una lucha por la existencia o una voluntad de vida es un impulso humano fundamental de aquellos que sienten la necesidad de distinguirse (Sorgner, 2007). Los seres humanos se esfuerzan por el poder. La lucha por la existencia representa solo un tipo marginal de la expresión fundamental de la voluntad de poder.

Si tienes voluntad de poder, entonces es de tu interés mejorarte a ti mismo. La mejora es precisamente el objetivo de los transhumanistas. El transhumanismo está a favor de las tecnologías y de otros medios que podrían utilizarse para la "mejora humana de aspectos intelectuales, físicos y capacidades emocionales" (Bostrom, 2001. http://www.nickbostrom.com/tra/values. html) para que los posthumanos puedan llegar a existir. En consecuencia, Bostrom recalca que los transhumanistas valoran un tipo de liberalismo que implica que las personas tienen el derecho a elegir "a vivir mucho más tiempo y con mejor salud, para mejorar la memoria y otras facultades intelectuales, para perfeccionar sus experiencias emocionales, así como la sensación subjetiva de bienestar, y en general, para lograr un mayor grado de control sobre sus propias vidas" (Bostrom, 2005b, p. 1). Bostrom evidentemente ha detallado esto más en relación con lo que todas estas demandas implican, así como con lo que no implican. Sin embargo, lo importante es que, en contraste con Habermas, valora la opción de que los padres puedan elegir la composición genética de sus hijos.

Habermas distingue entre los niños que simplemente se convirtieron en lo que son y los que se hicieron de una manera específica, y afirma lo siguiente:

10 Cf. Babich, 1994; Birx, 2006, vol. 4, pp. 1741-1745; Moore/Brobjer, 2004 
En primer lugar, el acto de imposición de los padres al elegir una composición genética, se supone que es inmoral, ya que se presume que los niños se sentirán forzados en cierta dirección, si su composición genética fue elegida por sus padres, más que si se convirtieron en lo que son por casualidad (2001, pp. 53-55).

En segundo lugar, se supone que hay una diferencia entre decidir la educación de los propios hijos y decidir acerca de su composición genética (Habermas, 2001). Los niños supuestamente son capaces de hacer algo en contra de la manera en que están siendo educados, además de que la educación promueve cualidades que luego pueden ser cambiadas (Habermas, 2001). Sin embargo, una composición genética no se puede cambiar, una vez que se tiene. Por lo tanto, según Habermas, la elección de una cierta composición genética para los niños propios y su educación son, moralmente hablando, dos tipos diferentes de hechos relativos a su evaluación moral.

Bostrom señala lo siguiente: "Los transhumanistas también sostienen que no hay ningún mérito ético especial en jugar a la ruleta genética. Dejar que la suerte determine la identidad genética de nuestros hijos nos ahorra directamente de una confrontación directa con algunas decisiones difíciles" (2001.http://www.nickbostrom.com/tra/values.html). Siendo esto así, simplemente rechaza el primer punto de Habermas. Parece estar implícito en su posición que la mayoría de los padres aman a sus hijos, de lo que se deduciría que la mayoría de los padres desean el bien de sus hijos. Ese "bien" puede ser algo que los padres consideran como bueno o algo que consideran bueno en función del interés de los niños. Sin embargo, no importa el concepto de bien que los padres prefieran, por lo general es mejor que los padres decidan el potencial genético del niño a que sea el resultado de una ruleta genética o un producto de la suerte. Es así como Bostrom argumenta que la segunda crítica de Habermas tampoco se sostiene. Si el diseño genético que los padres deciden es mejor en la mayoría de los casos que el que se recibe por casualidad, entonces, de forma evidente, moralmente no importa que no se pueda alterar después, al menos no tan fácilmente como las cualidades que se desarrollaron como resultado de la educación. Incluso se podría estar tentado a decir que, en la mayoría de los casos, es incluso mejor que estas cualidades no se puedan alterar, ya que son buenas para el niño. En este 
caso, también hay que señalar que no es nada claro que el segundo punto de Habermas sea correcto. Podría darse el caso de que muchas cualidades que se desarrollan sobre la base de la educación queden tan profundamente incrustadas en la propia personalidad que tampoco puedan ser alteradas de manera significativa.

Los críticos de la ingeniería genética también tienden a subrayar los peligros relacionados con los nuevos métodos tecnológicos: que algunas cosas ciertamente van a ir mal al principio y que no se debe jugar con los seres humanos, ni tratarlos solamente como un medio. Con respecto a tales preocupaciones, Bostrom responde:

El transhumanismo tiende hacia el pragmatismo (...) adoptar un enfoque constructivo, de resolución de problemas a los retos, favoreciendo los métodos que la experiencia nos dice que dan buenos resultados y tomar la iniciativa para "hacer algo al respecto" en lugar de solo quedarse quejándose (2001. http://www.nickbostrom.com/tra/values.html).

Él es directo, como todos los científicos y técnicos que tienen como objetivo nuevas metas y que, precisamente por nuevas, entran en aguas potencialmente peligrosas. Lo mismo se aplica a los investigadores en el campo de la ingeniería genética. América no habría sido descubierta ni tampoco se hubiera desarrollado la vacunación contra la viruela, si no hubiera existido gente lo suficientemente valiente para hacer lo que era esencial para el cumplimiento de esas tareas.

El valor es una virtud importante dentro de la moral de Nietzsche. También enfatiza la importancia de la ciencia para los próximos siglos y no rechaza su desarrollo. Teniendo en cuenta estas dos premisas, no se puede excluir la posibilidad de que Nietzsche habría estado a favor de la ingeniería genética, a pesar de que destaca la importancia de la educación para que se produzca el paso evolutivo hacia el sobrehumano. Si la ingeniería genética o la eugenesia liberal pueden ser realmente vistas como un tipo especial de educación (que es lo que los transhumanistas parecen sostener), entonces es posible que esta posición también se pueda mantener en Nietzsche, puesto que la educación juega un papel importante en su ética. Nietzsche hizo una 
afirmación de la ciencia, estaba a favor de la mejora y del advenimiento del sobrehumano.

\section{La perspectiva de los valores y el genio del Renacimiento.}

Los transhumanistas no tienen la intención de imponer sus valores sobre otras personas. Los "transhumanistas ponemos énfasis en la libertad individual y en la elección individual en el área de las tecnologías de mejora" (Bostrom, 2005b, p.11). Una de las razones para sostener esta posición es que Bostrom considera que es "un hecho que los seres humanos difieren ampliamente acerca de lo que consiste la propia perfección" (2001. http:// www.nickbostrom.com/tra/values.html). Y: "La segunda razón para este elemento del individualismo es el pobre historial en la toma colectiva de decisiones en el campo de las mejoras humanas. El movimiento eugenésico, por ejemplo, está desacreditado" (2001. http://www.nickbostrom.com/ tra/values.html). Bostrom utiliza aquí la palabra "eugenesia" pero se refiere solamente a un estado de eugenesia regulado, lo cual no considero que sea una forma útil de emplear esta noción (Sorgner, 2006). Él presenta una posición que podría ser llamada como una perspectiva de los valores. Este punto también es defendido por Nietzsche.

Cada constelación de poder y, por lo tanto, cada ser humano, según Nietzsche, tiene una perspectiva diferente sobre el mundo, y como cada concepción individual de poder depende de lo que uno es y de la historia que ha tenido, cada ser humano tiene un concepto único de poder y, en consecuencia, una concepción única "sobre lo que consiste su propia perfección". El mismo Nietzsche tiene un concepto claro de poder y lo que él considera como el más alto sentimiento de poder que está conectado directamente con el ideal clásico (Sorgner, 2007). Los transhumanistas parecen confirmar un ideal similar, según Bostrom:

El transhumanismo importa del humanismo secular el ideal de la personalidad plenamente desarrollada y bien redondeada. No todos podemos ser genios renacentistas, pero podemos tratar de refinarnos constantemente a nosotros mismos y ampliar nuestros horizontes intelectuales (2001. http:// www.nickbostrom.com/tra/values.html). 
No solo el aspecto de la "personalidad totalmente desarrollada y bien redondeada" se puede encontrar en Nietzsche, sino también el esfuerzo "para refinarnos constantemente a nosotros mismos y ampliar nuestros horizontes intelectuales". En Nietzsche (1967), este aspecto se denomina superación (overcoming). Los seres humanos superiores ${ }^{11}$ desean superarse permanentemente a sí mismos, para volverse más fuertes en los diversos aspectos que pueden ser desarrollados en un ser humano, para que finalmente el sobrehumano pueda existir. En el pensamiento transhumanista, el sobrehumano de Nietzsche se nombra como posthumano.

\section{El posthumano, el transhumano y el sobrehumano de Nietzsche.}

¿Quién es un posthumano? ¿Qué cualidades tiene? Creo que las únicas cualidades a las que todos los transhumanistas pueden suscribirse son las siguientes: "carecemos de la capacidad para formar una comprensión intuitiva realista de lo que sería ser un post-humano" (Bostrom, 2001. http://www. nickbostrom.com/tra/values.html). Sin embargo, varios transhumanistas han tratado de describir un posthumano con más detalle. Según Bostrom, F. M. Esfandiary (1989) sostuvo el siguiente concepto: "Un transhumano es un -humano en transición-alguien que, en virtud de su uso de la tecnología, los valores culturales y su estilo de vida constituye un eslabón evolutivo para la próxima era de la posthumanidad" (citado por Bostrom, 2005a, p. 12). En ese caso, un transhumano todavía pertenecería a la especie de los seres humanos, aunque en algunos aspectos ya habría desarrollado cualidades que extenderían el concepto de ser humano, al mismo tiempo que tendría el potencial de establecerse como la base para el paso evolutivo para una nueva especie. La nueva especie que representaría una nueva etapa de la evolución se conoce como los posthumanos. Por lo tanto, los transhumanos y los seres humanos tienen la capacidad de reproducirse entre sí, pero los

11 En las traducciones en castellano de las obras de Nietzsche usualmente se les nombra como los hombres superiores. En la mayoría de los casos, se refiere a ellos como artistas o creadores. Otras veces como espíritus nobles, a partir de un cierto ideal de aristocratismo social. No se refiere a algún tipo de "superioridad" racial, anatómica, biológica, ni ideológica en un sentido marxista. En algunos otros casos, Nietzsche también se refiere a ellos como los discípulos de Dionisios. En cuanto a esta última acepción, a pesar de conservar esencialmente la idea de lo que es un ser humano superior, no posee exactamente el mismo sentido, ya que parece que Nietzsche utiliza esta forma cuando intenta mencionar (en un sentido amplio) contextos más o menos políticos. Aun cuando no se refiera a líderes políticos, propiamente. N. del T. 
posthumanos no podrían hacerlo. De la misma manera que no podemos reproducirnos con los grandes simios, al menos no de una manera sexual. Incluso los posthumanos podrían depender de los medios tecnológicos para la reproducción.

El concepto del posthumano de Bostrom parece ser un poco diferente al de Esfandiary: "Por una capacidad post-humana, me refiero a una capacidad central en general muy superior a la máxima alcanzable por cualquier ser humano actual, sin recurrir a nuevos medios tecnológicos" (Bostrom, 2006, p. 1). Se hace evidente que las capacidades posthumanas no pueden ser idénticas a las cualidades que los actuales seres humanos tienen. Sin embargo, Bostrom $^{12}$ sigue pensando que podemos desarrollarnos en estos seres. Por lo tanto, no se refiere a nosotros como la especie de los seres humanos que pueden evolucionar hasta convertirse en una nueva especie con capacidades que son mucho más complejas que las nuestras, pero él piensa que cualquier ser humano, por medio de la tecnología u otros métodos, podría ser capaz de convertirse en un posthumano. Incluso afirma: "Esto podría hacer posible que se conserve la identidad personal durante la transformación en posthumano" (Bostrom, 2006, p. 15). Por eso, parece tener en cuenta que tanto los actuales seres humanos, como los posthumanos, pertenecen a la especie de los seres humanos, lo que quiere decir que tienen el potencial de reproducirse con otros medios distintos a la reproducción sexual. Los posthumanos no son una especie separada, sino un determinado grupo de seres humanos con capacidades que aún no pueden ser imaginadas por nosotros, pero que pueden implicar una mejora en todos los aspectos humanos, incluyendo una mejora fisiológica, emocional o intelectual. Bostrom sugiere que sería más probable para nosotros adquirir estas capacidades por medios tecnológicos. ${ }^{13}$

Permítanme aclarar algunas opciones de la mejora en general según Bostrom. Para esto voy a emplear el concepto de eugenesia de una forma que no lo hace Bostrom, pero que considero apropiada. ${ }^{14}$ Hemos tenido ejemplos de la eugenesia regulada por el Estado y la eugenesia liberal. La eugenesia regulada por el Estado es el tipo de eugenesia presente en el Tercer

12 "Supongamos que usted fuera a convertirse en un ser que tiene una esperanza de vida saludable (healthspan) posthumana y capacidades cognitvas y emocionales posthumanas" (Bostrom, 2006, p. 5).

13 "Podemos observar, sin embargo, que es poco probable que, en la práctica, que alguien pueda convertirse en post-humano por medio distinto a la tecnología avanzada" (Bostrom, 2006, p. 22).

14 Cf. Sorgner, 2007, pp. 53-58. 
Reich, la cual es moralmente despreciable y que en la actualidad la mayoría de los especialistas en ética occidentales que son serios, si no es que todos, la consideran como algo que debe ser evitado. La eugenesia liberal, por el contrario, se está discutiendo hoy en día como una posibilidad moralmente legítima, y académicos como Nicholas Agar (1998) están a favor de algunos actos asociados a la eugenesia liberal. Los transhumanistas, como se ha mencionado antes, también consideran a la eugenesia liberal como una manera moralmente legítima para la mejora de los seres humanos. Ahora bien, tanto la eugenesia regulada por el Estado como la eugenesia liberal son, no obstante, tipos heterónomos de eugenesia, lo que quiere decir que las personas deciden acerca de la mejora de otras personas. En el caso de la eugenesia regulada por el Estado, este es el que decide, mientras que en el caso de la eugenesia liberal son los padres quienes tienen el derecho a decidir sobre lo que se debe hacer con la descendencia. Los transhumanistas parecen identificar un tipo adicional de la eugenesia, la cual sugiero que sea llamada eugenesia autónoma. En esta las personas pueden decidir por sí mismas si desean ser transformadas en posthumanos por medios tecnológicos. Según como se presenta el tema en los artículos de Bostrom, esta parece ser la forma dominante en que se espera que los posthumanos, "un tipo extremadamente valioso" (Bostrom 2006, p. 24), lleguen a existir. ${ }^{15}$

Teniendo en cuenta el análisis anterior sobre las dos concepciones de posthumano, afirmo que los conceptos de seres humanos superiores y el sobrehumano de Nietzsche son muy similares a los conceptos del transhumano y el posthumano de Esfandiary, pero no así a los conceptos de Bostrom. Según Nietzsche, los miembros individuales de la especie de los seres humanos tienen la capacidad limitada de desarrollar solo ciertas cualidades. Se supone que es característico de todas las especies que sus respectivos miembros solo puedan desarrollarse dentro de los límites fijados. Si se dan ciertas condiciones, que Nietzsche no especifica, la evolución podría darse. Según Nietzsche, la evolución no es un desarrollo gradual de una especie a otra,

15 "De ello se deduce trivialmente de la definición de -post-humano- que figura en este documento que no somos posthumanos en el momento que estamos escribiendo. No se sigue, al menos no de una manera obvia, que un post-humano no podría seguir siendo también un ser humano. Aunque que esto sea así o no, depende del significado que le asignamos a la palabra -humano-. Bien se podría tener una visión amplia de lo que significa ser humano, en cuyo caso -posthumano- ha de entenderse como denotando un cierto modo posible de ser humano -. Si estoy en lo cierto, un tipo muy valioso" (Bostrom, 2006, p. 24). 
sino que se da por pasos. Si las condiciones dentro de una especie son tales que un paso evolutivo puede darse, varias parejas al mismo tiempo podrían dar a luz a miembros de una nueva especie. Las parejas que darían a luz a los sobrehumanos deberían tener cualidades a las que Nietzsche se refiere como las que poseen los seres humanos superiores. Una de las condiciones necesarias para que se dé un paso evolutivo es que existan muchos seres humanos superiores. Normalmente, un ser humano superior no puede simplemente transferir sus capacidades excepcionales a sus descendientes. Sin embargo, si hay muchos seres humanos superiores y se presentan, además, algunas otras condiciones, un paso evolutivo podría ocurrir (Nietzsche, 1967).

Los seres humanos superiores todavía pertenecen a la especie humana, pero tienen algunas capacidades especiales que un sobrehumano también podría tener. No obstante, los seres humanos superiores no pueden transmitir sus capacidades especiales a sus descendientes por medio de la reproducción sexual. Es por casualidad que los seres humanos superiores tienen el potencial que tienen y, además, tienen que poner un esfuerzo significativo para el desarrollo de sus diversas capacidades. Según Nietzsche (1967), Goethe representa el mejor ejemplo de un ser humano superior. Los seres humanos superiores que Nietzsche menciona se basan en un carácter especial que tienen por casualidad. Su naturaleza les permite desarrollarse como seres humanos superiores, si trabajan duro para mejorarse a sí mismos. Por eso, hace hincapié especial en el desarrollo de las capacidades intelectuales, sobre todo en la habilidad de interpretar. Nietzsche no se refiere a los medios tecnológicos de mejora -en este sentido Bostrom acierta-, sin embargo, tampoco excluye la posibilidad adicional de la mejora tecnológica.

El sobrehumano tiene un potencial significativamente diferente al de los seres humanos superiores. Hasta el momento no ha existido ningún sobrehumano, pero las capacidades normales de un sobrehumano están más allá de las capacidades incluso de un ser humano superior. Al igual que todas las especies, la especie del sobrehumano tiene límites, pero sus limitaciones son diferentes a las de la especie humana. El sobrehumano tiene lugar a través de un paso evolutivo que se origina en el grupo de los seres humanos superiores. Como dije, Nietzsche no excluye la posibilidad de que los medios tecnológicos lleven a cabo esta etapa evolutiva. Sus comentarios relativos a las condiciones para el paso evolutivo hacia el sobrehumano son 
bastante vagos en general, pero en este aspecto su actitud es similar a la de los transhumanistas. Sin embargo, él cree que el espíritu científico gobernará los próximos milenios y que este espíritu traerá consigo el final de la dominación de los conceptos dualistas de Dios y la metafísica, por lo que esto hará ampliamente plausible su pensamiento.

Teniendo en cuenta esta breve caracterización de los seres humanos superiores, así como la del sobrehumano, estoy obligado a concluir que los seres humanos superiores son similares al concepto del transhumano de Esfandiary y que el sobrehumano de Nietzsche abraza muchas similitudes con el posthumano también de Esfandiary. Ahora, ¿qué podemos decir sobre el concepto de Bostrom del posthumano si lo comparamos con los conceptos de Nietzsche?

Bostrom sostiene: "Uno bien podría tener una visión amplia de lo que significa ser humano, en cuyo caso - posthumano- debe de entenderse como denotando un cierto tipo posible de modo de ser humano" (2006, p. 24). De acuerdo con esto, también sostiene que los posthumanos tienen capacidades que no se pueden encontrar en los seres humanos vivos. Como Nietzsche defiende que la especie de los seres humanos tiene límites estrictos, es bastante improbable que el concepto de un tipo de ser humano con capacidades que aún no han existido sea coherente con su filosofía. En consecuencia, se puede concluir que Nietzsche y los transhumanistas comparten muchos aspectos de manera general en sus antropologías y sus valores, aunque el concepto de sobrehumano de Nietzsche no corresponde con el concepto del posthumano de todos los transhumanistas. Sin embargo, hay transhumanistas cuyo concepto del posthumano tiene muchas similitudes significativas con el de sobrehumano de Nietzsche.

\section{El sobrehumano y la esperanza de Nietzsche para el futuro}

Los transhumanistas, al menos en los artículos que he consultado, no han explicado por qué tienen los valores que tienen, ni por qué debería sobrevenir el posthumano. Nietzsche, por su parte, sí explica la relevancia del sobrehumano para su filosofía. El sobrehumano puede, incluso, ser el fundamento último de su visión de mundo. 
Nietzsche ve a los filósofos como creadores de valores, esto se fundamenta, en última instancia, en sus prejuicios personales. ${ }^{16}$ Él se refiere a sus propios prejuicios como aquellos que corresponden con el espíritu que gobernará los próximos siglos. Espíritu aquí no se refiere a un nous inmaterial en el sentido platónico o a alguna sustancia espiritual. Espíritu, en los escritos de Nietzsche, quiere decir una capacidad corporal de interpretación que se da por el lenguaje y que se basa en la fuerza fisiológica. Distingue, además, entre un espíritu religioso y un espíritu científico. Los seres humanos reactivos y débiles que no pueden cumplir con sus deseos en el aquí y ahora, incorporan el espíritu religioso, lo que les crea un anhelo para quienes desean una buena vida en el más allá. Este espíritu fue dominante entre los seres humanos por un período muy largo. Sin embargo, con el tiempo, los seres humanos se hicieron más fuertes, lo que consecuentemente hizo que se desarrollara cada vez más un espíritu científico. La importancia del espíritu científico se ha incrementado significativamente, sobre todo desde el Renacimiento. Nietzsche espera que este espíritu se vuelva aún más dominante en el futuro. Como supone que su visión del mundo es atrayente para el espíritu científico, presume que cada vez será más atractiva para la gente del futuro.

Según Nietzsche, Platón puede ser visto como un representante de una filosofía basada en el espíritu religioso, mientras que él se puede ver como un representante de una filosofía basada en el científico. El cristianismo, que fue dominante en los países occidentales desde hace mucho tiempo, se debe considerar como el platonismo para el pueblo. Es una necesidad de Nietzsche, así como su intención, voltear este platonismo. De hecho, se refiere a su propia filosofía como un platonismo invertido. De la misma manera, como el pensamiento cristiano ha dominado muchos siglos, su modo de pensar científico se conjetura para gobernar los próximos siglos. En consecuencia, las versiones invertidas de los principales elementos del pensamiento platónico-cristiano se encuentran en el pensamiento de Nietzsche.

Un aspecto central del cristianismo, según Nietzsche, es una vida personal en el más allá. Es lo que hace que el pensamiento cristiano sea atractivo para muchas personas, al mismo tiempo que les da un sentido a sus vidas. Si mi representación del pensamiento de Nietzsche es correcta,

16 Los siguientes párrafos son un resumen de mi lectura de Nietzsche que se explica en detalle en mi monografía Metaphysics without Truth (2007). 
entonces una versión invertida de esta vida futura personal en el más allá también tiene que ser parte del pensamiento de Nietzsche o al menos debe haber un concepto que le brinde un sentido a la vida de los seres humanos. Es aquí donde entra el sobrehumano, junto con otro concepto: el eterno retorno -la teoría de la salvación de este mundo de Nietzsche- que no voy a tratar en este texto, a pesar de que todos estos conceptos están estrechamente relacionados entre sí.

El sobrehumano simboliza el sentido de la tierra. El sobrehumano, supuestamente, representa la concepción que da un significado (meaning-giving) existencial dentro de la visión del mundo de Nietzsche, para reemplazar la visión del mundo, básicamente cristiana. Este se presenta dentro del interés de los seres humanos superiores para superarse constantemente. El tipo final de superación se puede ver en la superación de la especie humana y quien haya tenido mucho interés en superarse permanentemente puede considerarse a sí mismo como un antepasado del sobrehumano. Es de esta manera como se supone que el sobrehumano da sentido a los seres humanos. No es un sentido trascendente, sino uno terrenal, inmanente, que es más apropiado para las personas de mentalidad científica que han abandonado su creencia en un mundo del más allá. Como subraya C. G. Jung: "El hombre no puede soportar una vida sin sentido" (Stevens, 1994, p. 126). Nietzsche y Platón estarían de acuerdo. Sospecho que el concepto transhumanista del posthumano no se puede apreciar plenamente, si no se tiene en cuenta este aspecto que da un sentido existencial o si se quiere excluir todas las referencias a conceptos cuasi religiosos. Bostrom (2006) en un contexto diferente invoca lo siguiente:

Muchas personas que tienen creencias religiosas ya están acostumbrados a la perspectiva de una transformación muy radical en una especie de ser posthumano, que se espera que tenga lugar después de la terminación de su encarnación física actual. La mayoría de los que sostienen este punto de vista también sostienen que la transformación podría ser muy buena para la persona que se transforma (pp. 16-17).

Sospecho que el valor del advenimiento del posthumano no se puede justificar en última instancia, excepto cuando un individuo cree que esta 
concepción hace su vida significativa: "Deseo ser el ancestro de un posthumano". Dudo de que Bostrom esté de acuerdo con esta sospecha, ya que se podría temer una mezcla de categorías científicas y religiosas. Yo, por el contrario, creo que el concepto del posthumano se puede hacer más fuerte, si se acepta que también tiene una función que implica brindar un sentido existencial que, a diferencia de la vida del más allá cristiano, se basa en esperanzas científicas, en la importancia del mundo de los sentidos y de objetivos inmanentes. Nietzsche confirmó que el concepto de sobrehumano es el sentido de la Tierra. Creo que la importancia del posthumano solo puede apreciarse plenamente si uno reconoce que su fundamento último es que brinda un sentido a la gente de mentalidad científica. No creo que haya nada malo o abominable en eso.

\section{Referencias}

Agar, N. (1998). Liberal eugenics. Public Affairs Quarterly, 12(2), 137-155.

Ansell-Pearson, K. (1997). Viroid life: Perspectives on Nietzsche and the transhuman condition. London: Routledge.

Babich, B. E. (1994). Nietzsche's philosophy of science. Albany: State University of New York Press.

Birx, H. J. (2006). Nietzsche. In H. J. Birx (ed.), Encyclopedia of anthropology. 5 vol., Thousand Oaks, California: SAGE Publications., 1741-1745

Bostrom, N. (2001). Transhumanist values. Recuperado de http://www.nickbostrom.com/ tr a/values.html

Bostrom, N. (2005a). A history of transhumanist thought. Journal of Evolution and Technology, 14(1), 1-30.

Bostrom, N. (2005b). Transhumanist values. Review of Contemporary Philosophy, 4, 3-14. https://doi.org/10.5840/jpr_2005_26

Bostrom, N. (2006). Why I want to be a Posthuman when I grow up. Medical enhancement and posthumanity, 1, 107-137.

Habermas, J. (2001). Die Zukunft der menschlichen Natur. Auf dem Weg zu einer liberalen Eugenik? Frankfurt am Main: Suhrkamp.

Nietzsche, F. (1967). Sämtliche Werke. Kritische Studienausgabe in 15 Bänden. Hg. v. G. Colli u. M. Montinari, München. New York: Deutscher Taschenbuch Verlag.

Sorgner, S. L. (2007). Metaphysics without truth. On the importance of consistency within Nietzsche's philosophy. Revised edition. Milwaukee, WI: University of Marquette Press.

Sorgner, S. L. (2006). Facetten der Eugenik. In S.L. Sorgner, H. J. Birx and N. Knoepffler (eds.), Eugenik und die Zukunft. Feiburg i. B.: Alber Verlag, (P. 201-209).

Stevens, A. 1994. Jung. Oxford: Oxford University Press. 